www.elsevier.com/locate/shpsc

\title{
In Defence of Classification
}

\author{
John Dupré*
}

It has increasingly been recognised that units of biological classification cannot be identified with the units of evolution. After briefly defending the necessity of this distinction I argue, contrary to the prevailing orthodoxy, that species should be treated as the fundamental units of classification and not, therefore, as units of evolution. This perspective fits well with the increasing tendency to reject the search for a monistic basis of classification and embrace a pluralistic and pragmatic account of the species category. It also provides a diagnosis of the paradoxical but popular idea that species are individuals: Species are not individuals, but the units of evolution are. (C) 2001 Elsevier Science Ltd. All rights reserved.

Keywords: Species; Classification; Taxonomy; Evolution; Darwin.

\section{Introduction}

A topic surprisingly little discussed in recent controversies about the nature of species is classification. By that I mean the practical activity of assigning the vast numbers of organisms in the world to particular kinds. One reason for this neglect, perhaps, is that writers on species are often theorists rather than the practitioners who actually classify organisms; and perhaps in some cases these two personae coexist in the same human body without communicating a great deal with one another. Or perhaps it is because many writers on this topic have reached the conclusion that species aren't kinds at all, but individuals, so that the nature of species can have little to do with the activity of classification. At any rate, it has become common to treat species primarily as units of evolution rather than units of classification: an influential recent anthology on the topic bears the title 'The Units of Evolution' and the subtitle 'Essays on the Nature of Species' (Ereshefsky, 1992).

It is of course possible that the units of evolution could also serve as units of classification, and that this is in fact the case seems to be very widely assumed by writers on the topic. But, and this is the first thesis I shall defend in this paper,

\footnotetext{
* Department of Sociology, Amory Building, University of Exeter, Exeter EX4 4RJ, U.K. Received 18 October 1999; in revised form 2 December 2000.
} 
units of evolution are far too diverse a set of entities to provide a useable partition of organisms into classificatory units. If this is correct we need to decide whether the term species should be applied to units of evolution or units of classification. And here, if anywhere in biology, it seems to me we should honour conventions of priority. Species were understood to be basal units of a classificatory system millennia before anyone had thought to formulate a theory of evolution. And to this day almost everyone who is not professionally involved in theoretical systematics assumes that the species category is a classificatory concept. So I conclude that species should be treated as units of classification not units of evolution.

As arguments for treating species as units of evolution are generally concerned to stress, evolution is the theory that dominates contemporary biology. As the frequently quoted title of a paper by Theodosius Dobzhansky (1973) puts it, 'Nothing in biology makes sense except in the light of evolution'. ${ }^{1}$ Sometimes this seems to be taken as sufficient grounds for concluding that species must be the units of evolution. We do, after all, generally classify the objects with which a scientific theory deals in relation to the demands of that theory. But this argument, even when more thoroughly fleshed out, is unconvincing. Classification in biology has a life of its own. Biologists in areas only tangentially connected to evolutionary theory, such as ecologists, ethnobotanists, or ethologists, need to classify organisms, as do foresters, conservationists, gamekeepers, and herbalists. As will be discussed below, for many, perhaps even most groups of organisms, evolutionary considerations are of little or no use for classificatory purposes. And finally birdwatchers, wildflower enthusiasts, or just biologically engaged members of the public, may choose to classify organisms, even if they do not need to do so. These diverse groups of people require workable classifications that enable them to communicate among themselves and to members of other such groups, record information about natural history, and so on. If, as I argue, units of evolution inadequately meet these needs they must be distinguished from units of classification. Furthermore, it follows that in biology classification and theory are more autonomous from one another than seems to be the case in most parts of science. The fact that classification cannot, at least, be closely tied to the central theory of biology leaves room for the thoroughly pragmatic and pluralistic approach to biological taxonomy that I shall advocate.

I have mentioned the thesis that species are individuals. This thesis is based on compelling arguments that the units of evolution are individuals. If it were possible to equate the units of evolution with the units of classification then it might be legitimate to conclude from this, somewhat paradoxically, that the units of classification were individuals. But since this is not possible, there is no temptation to embrace this paradoxical conclusion. There are, on the other hand, various concepts

\footnotetext{
${ }^{1}$ Actually I suspect that much of molecular biology would make perfectly good sense if biological organisms had been deliberately designed by God or Martians. And arguably molecular biology is currently a rather more active field (though not, perhaps, a theory) than is the study of evolution.
} 
more explicitly tied to the theory of evolution that refer to groups of organisms, for example 'population', 'lineage' and 'clade'. The arguments that species are individuals show unproblematically that we should treat these terms as applying to individuals. These points will be developed in more detail later in the paper.

\section{Units of Evolution and Units of Classification}

I have referred above to the question of whether units of evolution and units of classification might be identified with one another. But if, as I have acknowledged, the units of evolution are individuals, and since, as seems no more than a matter of definition, units of classification must be kinds, it is not at all clear what such an identification could mean. What is certainly possible is that one set of organisms could simultaneously constitute all and only the members of a kind, and all and only the parts of an individual. As a matter of fact I take it that this is often the case: the members of a species do often constitute a unit of evolution. ${ }^{2}$ But putting matters this way should also make it clear that once we distinguish these two kinds of units, and recognise that they belong to quite distinct ontological categories, it would be strange to insist that such coincidence must always occur. If the sets consisting of those organisms that were part of a distinct unit of evolution in fact turned out to serve well as kinds from the point of view of biological classification, then it might be very convenient to make such coincidence a rule of classificatory practice. But, as I shall now explain, units of evolution are often thoroughly illsuited for this additional task. ${ }^{3}$

What are units of evolution? What kind of entities, that is to say, evolve? Individual organisms develop, but are not said to evolve. Evolution occurs when the properties characteristic of the individuals in a phylogenetic lineage change over time, so we can say that the units of evolution are lineages of some kind. The problem, then, is which lineages are units of evolution. A natural suggestion is that to be units of evolution lineages must be coherent. We don't consider humans and chimpanzees to be part of the same unit of evolution, because we believe that these two groups are now evolving independently of one another. Thus primates, or mammals, do not appear to be units of evolution. At this point it is easy to see the appeal of the so-called Biological Species Concept (BSC). According to the BSC, a species is a group of organisms connected by reproductive relations and reproductively isolated from all other organisms. These conditions provide a satisfying account of the coherence of a lineage, and motivate the idea that the division of a lineage into two occurs when reproductive isolation is established between two parts of a lineage, paradigmatically between the main body of a species and a peripheral, geographically isolated, population.

\footnotetext{
${ }^{2}$ Strictly, such identities are probably only approximate, as questions about the limits of a species and about the origins of a lineage may be answered differently.

${ }^{3}$ A more detailed argument to this effect is developed in Dupré (1999).
} 
The BSC dominated systematic theory in the early years of the Modern Synthesis, and provided a tight conceptual link between evolutionary theory and taxonomy. Unfortunately in the last few years its limitations have become increasingly clear. As was recognised from its early days, it provides no account of the taxonomy of asexual organisms, and hence of all organisms for the greater part of the history of terrestrial life. Since asexual organisms certainly evolve and certainly require classification, this is a far more serious obstacle to the project of unifying evolutionary theory and taxonomy than has often been acknowledged. But even restricting our attention for the moment to sexually reproducing organisms there are very serious problems with the BSC. Recent work suggests that reproductive isolation is neither necessary nor sufficient for the existence of morphologically coherent lineages. The classic illustration of this is found in American oaks (genus Quercus). Here it seems that distinct morphological species have coexisted for substantial periods of evolutionary time while undergoing significant genetic exchange through hybridisation (Van Valen, 1976). ${ }^{4}$ It is of course possible to respond to this difficulty by saying that this merely shows that these trees constitute one morphologically diverse species. But this illustrates the problem that I shall argue recurs again and again in the marriage of theory and taxonomy: Theoretical considerations mandate a clearly suboptimal taxonomy. To the ecologist, forester or even landscape gardener, the kinds in question are naturally and appropriately treated as distinct species. ${ }^{5}$ A somewhat different kind of problem arises with large groups of organisms in which there are few if any sharp discontinuities, either reproductive or morphological. An example is the genus Rubus, the brambles and their relatives, which is distributed over most of the Earth. Here there may be no theoretical objection to treating such a group as a single enormous and morphologically diverse species. But given the morphological diversity there are surely pragmatic reasons for applying some kind of classification. The greengrocer and the patissier, at least, will want to distinguish the blackberry, the loganberry and the raspberry, not to mention many other, more obscure, named fruits.

The insufficiency of reproductive isolation to account for the distinctness of species is illustrated by the fact that morphologically homogeneous species often seem to exhibit surprisingly little genetic interchange (Ehrlich and Raven, 1969). In fact species often exist as numerous populations isolated from one another by impassable geographical obstacles, and yet appear to maintain their morphological

\footnotetext{
${ }^{4}$ Mayr (1996, p. 265) notes this difficulty, and suggests that it is sufficient to require only that there are properties of individuals that will prevent complete fusion of the populations. But this, surely, requires a criterion for distinguishing fused and non-fused populations, a criterion that will do all the work in deciding whether or not there is a real division between species. Magnus (1998) describes a hypothetical process by which speciation may occur sympatrically, suggesting that reproductive isolation is necessary neither for the continued coherence of a species, nor even for its origin.

${ }^{5}$ Stebbins (1987, p. 198) notes that even more traditionally construed species often provide too coarse a classification for foresters, as these may include trees with quite different ecological properties and requirements. It is interesting to compare Sterelny's (1999) suggestion that the term 'species' be reserved for metapopulations of populations occupying ecologically diverse situations.
} 
integrity. This suggests that genetic interchange is only one among a range of possible causes of the coherence of a species (see Templeton, 1989), once again emphasising that reproductive isolation is unnecessary. Of course the existence of such additional mechanisms is also indicated by cases such as the oaks, in which coherence appears to continue despite genetic introgression. The case of isolated populations introduces another important consideration. While there is no doubt that geographical isolation in some cases leads to the inception of a new distinct lineage, it may also be followed by reintegration with the main body of the species. Even if the isolated population has for a time undergone distinct evolution, the barriers to genetic exchange may be removed before the evolution of any feature that would provide a barrier to sympatric reproduction. ${ }^{6}$ Hence what is at one time a coherent and distinct unit of evolution may merge with another unit of evolution. Units of evolution do not form a tree-like structure, but are at least partially reticulated. Since evolutionarily grounded taxonomies are invariably presented in an unreticulated tree-like structure, this means that the question of whether a unit of evolution counts as coherent depends on the question of whether it will continue to evolve independently, or eventually remerge with the parent species. It is thus a question that in many cases will be operationally undecidable. It hardly needs emphasising that this is a disadvantage for a practically applicable taxonomy. A final point is that having recognised the likelihood that there are mechanisms other than reproductive isolation that can produce evolutionary coherence, it is entirely possible that there may be subspecific units of evolution deriving coherence from such mechanisms even when these are sympatric with the remainder of the species.

Two general problems emerge. First, if we attempt to apply a rigid criterion such as reproductive isolation we will end up with species which range in size from enormous groups of (currently recognised) species such as the brambles or the oaks, to groups consisting of a handful of organisms. Second, we begin to see that the very idea of a unit of evolution is much vaguer than might first have been supposed. The concept of 'reproductive isolation' suggests the picture of evolutionary change flowing down sharply defined channels, branching at well-defined nodes - and naturally identifies units of evolution as lengths of channel terminating at nodes. A more realistic picture would be a river estuary at low tide. We find large streams of water and many side streams, some petering out, others rejoining a main channel or crossing into a different channel, and a few maintaining their integrity to the ocean; there are islands around which streams flow and then rejoin; eddies and vortices; and so on. Some parts of the general flow are naturally and coherently distinguishable, and it is easy enough to recognise parts of the pattern that are definitely not parts of the same 'unit of flow'. But in between, there are

\footnotetext{
${ }^{6}$ Another way of looking at this case would be to suggest that reproductive isolation might indeed be a sufficient condition to constitute a unit of evolution. However, it would be highly undesirable to be committed to calling every temporarily isolated part of a species a distinct species, thus showing again that species should not be identified with units of evolution.
} 
many cases where any such distinction into discrete units would be largely arbitrary. To quote a prominent defender of an evolutionary grounded view of the species:

most species are more like slime molds and sponges than like highly organized and tightly integrated multicellular organisms - at least in terms of their individuality. Not only can almost any part of a species give rise to a new lineage, but those new lineages also commonly reunite after separating. Consequently there will be many cases in which it will be difficult to determine the precise number and boundaries of species ... (de Queiroz, 1999, p. 79).

The moral de Queiroz draws from this point is that it should no more lead us to doubt the reality of species than similar problems about the individuation of organisms should lead us to doubt the reality of organisms. I agree, provided that what is under discussion is the existence of lineages, or units of evolution. Whether the situation described is likely to provide us with a suitable basis for a classificatory system is quite another matter.

I introduced the present discussion by noting that a standard approach to these questions is to take it as a matter of definition that species are what evolve, and thus to take the answer to the question what lineages are the units of evolution as also providing the answer to the question what groups of organisms are species. What I hope to have shown is that this approach is probably unworkable, and to the extent that it is workable, will lead to a taxonomy that is seriously at odds with the desiderata of usefully sorting organisms into kinds in ways that will serve to convey and store information about the biological world.

One final worry about the divorce of species from units of evolution is not so much an argument as a matter of historical veneration. Darwin, the most venerable figure in the history of biology, did, after all, write a rather well known book on the origin of species, and convinced most of those worth convincing that the origin of species was that they evolved. So is the denial that species are what evolve not flying in the face of Darwin? In response to this I would say, first, that I am confident that my argument here contradicts nothing of the substance of Darwin's contribution. The greatest part of that contribution, I suppose, was the provision of a naturalistic explanation of adaptation, something almost wholly irrelevant to the present topic. He also had much to say about the origin of diversity, much closer to the present topic. But as far as I can see my main disagreement here is semantic: I don't disagree about the kind of things that evolved, only whether these should be referred to as species. Although, as I discuss in a later section of this paper (section 5), I do not take semantic debates to be trivial, I do suggest that this semantic dissent falls well short of sacrilege. In the conclusion of the paper I shall, as is customary in theoretical discussions of biology, suggest that the position I take is ultimately more faithful to the spirit of Darwin than are the positions I oppose.

I have not tried to discuss in detail the many specific proposals for evolutionarily 
grounded species concepts that have been developed as successors to the BSC, though I have discussed some of the most prominent elsewhere (Dupré, 1999). Here I mean only to give a general sense of why I think such projects are basically misguided. I now turn to a consideration of an alternative response to this situation.

\section{Pluralism About Species and The Aims of Classification}

I have no wish to deny that concordance with phylogeny is one desideratum in the provision of a taxonomy. In many of the phyla of greatest immediate interest to us, mammals or birds, for instance, it is probably reasonable to expect that taxonomy should recapitulate phylogeny. These are the taxa in which species are, in the main, more 'like highly organized and tightly integrated multicellular organisms'. Where such recapitulation is possible we should expect that evolutionary species should coincide with morphological distinctness. Michael Ruse has very optimistically claimed that 'the different ways of breaking organisms into groups ... coincide! The genetic species is the morphological species is the reproductively isolated species is the group with common ancestors' (Ruse, 1987, p. 237). No doubt this is often the case with these relatively well-behaved taxa. But it is surely not the case for most of the biological world. Hence I take this concordance to be a desideratum, but only one desideratum among several, and by no means the universally decisive one.

Once it is recognised that there is no theoretical grounding for a classificatory system that will universally or even generally provide a practically applicable taxonomy, we are free to embrace taxonomic pluralism. Approaches to classification will vary from one group of organisms to another, and we should allow experts in particular phyla to decide the most appropriate way of classifying a particular domain. It is worthwhile, however, to insist on the importance of certain desiderata derived from considerations of the nature and function of classification itself, an insistence that only becomes possible once we are freed from the prejudice that the ultimate criteria of classificatory excellence must be imported from some external source, evolutionary theory. In many parts of biology, for example bacteria and many orders of flowering plants, it is doubtful whether any evolutionarily grounded taxonomic scheme will be feasible, and it may be necessary to resort to morphology. This does not, of course, mean that we must adopt the discredited pheneticist notion of absolute similarity. Morphological features must be selected for taxonomic purposes, perhaps on the basis of biological salience (as Linnaeus considered the sexual parts of flowering parts to have particular biological importance) or perhaps just on the basis of suitability for taxonomic purposes themselves. This leads us again to the question of what the intrinsic virtues of a taxonomic scheme should be. Some obvious ones can readily be identified: 


\section{(a) Comprehensiveness}

A requirement of an adequate system of classification is that everything within its domain - in the present case every organism — should be included within it. This does not of course preclude the possibility that some allocations of organisms to taxa will be more or less arbitrary; but in that case there will at least be an approximate boundary between kinds that determines some possible decisions about the kind to which any organism belongs. There might perhaps be no fact of the matter whether I am looking at an instance of Rudbeckia hirta or of Rudbeckia triloba; or whether, perhaps, it is some kind of hybrid between the two. But it certainly isn't a fish, or even an oak tree. And if I tell you it's a Black-eyed Susan you shouldn't be seriously misled. The condition that something useful can be said about any organism seems a minimal requirement for an acceptable taxonomy. ${ }^{7}$

\section{(b) Suitably sized base-level groups}

One of the main defects with reproductively or phylogenetically determined groups is that these tend to generate groups differing in size by many orders of magnitude. This defect can perhaps be remedied by the kind of moderate pluralism proposed by Mishler and Donoghue (1982), who insist that species should be monophyletic, while adopting a pluralistic attitude to the question of what monophyletic units should count as species. This remedy may make one wonder, however, what virtue is being served by the continued insistence on monophyly. The criterion of suitable size is necessarily a vague one. Bitterns are rarer than blackbirds, but this should not motivate the attempt to divide blackbirds up into lots of geographic races about as numerous as are bitterns. Despite this vagueness, however, it is clear enough that some such desideratum is violated by the suggestion that there is only one species of deciduous oak in America, or that common types of bacteria should be divided into innumerable species the vast majority of which go extinct long before anyone bothers to distinguish them.

\section{(c) Reasonably homogeneous groups}

and

\section{(d) Reasonably sharp boundaries between groups}

I treat these together because the limits to their attainability can be said jointly to define the problem for biological taxonomy, and the respect in which it differs from traditional essentialist taxonomy. An ideal taxonomy would distinguish entities as members of wholly homogeneous classes, which are reliably distinguishable from the equally homogeneous members of every other class. This ideal would have been achieved by a taxonomy based on the essential property or properties

\footnotetext{
${ }^{7}$ Sterelny (1999), however, argues that only some kinds of organisms are organised into species. I take this as illustrating my claim that species thus conceived are inadequate as a basis for taxonomy. Sterelny's position is discussed briefly below.
} 
of members of the taxon, if only there had been such properties. We know that the phenomena of biological variation, hybridisation, and more or less imperfect separation between clades make such a taxonomy unachievable in biology. But the best must not be allowed to be the enemy of the good. These characteristics continue to remain ideals of classification, simply because in obvious ways they make taxonomies easier to use. The ideal of homogeneity is another part of the reason why groups such as the oaks and blackberries do not provide good base level taxa. Unlike homogeneity sharp boundaries are always attainable. In many familiar cases they simply exist between fully isolated and phylogenetically distinct groups and can be identified through several or many different possible criteria. But where this is not the case they can still be defined. One can, for example, distinguish blackberries as belonging to a particular species by drawing geographic lines and defining anything to the south of the line as belonging to one species, anything to the north to another. Whether such a consequence as different parts of the same bush belonging to different species would be a price worth paying for this advantage is another matter.

\section{(e) Taxonomic conservatism}

A quite different kind of desideratum for a taxonomic scheme is that it should be as stable as possible. Consumers of taxonomy, people who use the results of expert taxonomists in their everyday interactions with the biological environment, often complain about the constant tendency of biologists to change classifications, thus making existing taxonomic expertise ineffective. The main relevance of this to the present topic is the argument it provides, from the perspective of a pragmatically useful taxonomy, against the assumption that taxonomy should be theorydriven. The nature of species is a notoriously contentious matter, so it is hardly surprising that biologists driven by one conception of the species should feel compelled to revise the taxonomies of their predecessors, often from a very different theoretical perspective. More important still, commitment to a uniform theoretical view of the species category implies that new biological information will constantly motivate changes to taxonomy. If, for example, one is committed to the Biological Species Concept, then the discovery that particular populations do or do not interbreed will force revisions of the application of species names. The advantages of taxonomic stability are obvious and considerable, maximising the stability of detailed biological knowledge whether stored in libraries or human brains. The fact that a pragmatic and pluralistic conception of taxonomy is able to set a much higher standard for taxonomic revision is a considerable advantage for such an approach.

I have not, of course, said anything about the biological rationale for particular conceptions of taxonomy. To the extent that a biological rationale is seen as forcing taxonomy into the theoretically sanctioned form of monism, any such rationale is opposed to the position I am defending. However, it is quite consistent with my view that theoretical biological considerations should be locally important in 
determining how particular groups of organisms should be classified. As noted above, it is a fine thing if a taxonomy can coincide with a plausible phylogeny. For groups such as birds and mammals this is a realistic goal, and may even be a reasonable constraint on taxonomic practice. However it is almost certainly not a generally feasible constraint. It almost certainly cannot be applied to prokaryotes, hence to the first half of evolutionary history, and perhaps is only applicable to a few extremely highly evolved and sophisticated groups of metazoans. The only possible unifying conception of the species is as a unit not of evolution but of classification.

This is the view of species that I propose. Species are a unified category only in the very weak sense that they are (by definition, I suggest) the basal units of classification. But the criteria used for distinguishing species from one another, and the theoretical rationale, if any, for such distinctions, should be allowed to vary promiscuously from one taxon to another.

\section{Units of Evolution as Individuals}

One of the most widely discussed theses in the recent philosophy of biology is the idea that species are individuals. ${ }^{8}$ Provided it is assumed that species are units of evolution, this thesis is extremely compelling. To begin with the most obvious and, I believe, the fundamental point, evolution is a process that something, presumably, undergoes. But it is doubtful whether it even makes sense to speak of a kind undergoing such a process. A kind can be the object of mental processes; it can be contemplated or its members can be counted. But it isn't the kind of thing that can undergo processes in the world outside. It is true that collectives consisting of numbers of individuals can undergo processes. A crowd can become unruly, or a herd of cows stampede. But of course a crowd or a herd is not a kind. One is a member of a crowd by virtue of the relations (mainly spatial) that one bears to other members, not by virtue of any intrinsic properties one may have. The possible, if unlikely, crowds in Star Wars movies consist of exceedingly diverse entities; and the identical twin of a member of a crowd is not, by virtue of similarity, a member of the same crowd. Moreover, from the point of view of the theory of crowds, crowds are individuals, and members of crowds are parts of those individuals. It is extremely plausible that the same should be said for species qua units of evolution. If, somehow, the kind Horse were evolving, then possible horses in distant galaxies would be participating in the same process as would merely terrestrial horses. For presumably terrestrial and extragalactic horses bear the same relation to the kind Horse. But of course this is nonsense, and the evolution of horses on Earth has no bearing on whatever horses there may be elsewhere in the universe. An obvious explanation is that horses are parts of the evolving unit rather

\footnotetext{
${ }^{8}$ The most detailed elaboration of this idea is by Michael Ghiselin (1997), who first introduced the thesis in the 1960 s.
} 
than members of an evolving kind. And it is individuals, never kinds, that have parts.

Another commonly cited argument is that kinds, or at any rate natural kindswhich species would presumably be if they were kinds-are the subjects of laws of nature; and there are no laws, it is said, about species. I am rather less sure about this argument, if only because it seems to me so far from clear what a law of nature is. Many laws of nature appear to be probabilistic, and the fact that there is always or almost always variation among the members of a species does not obviously preclude there being probabilistic laws about such members. Matters are murkier if we take it for granted that the question is whether there could be laws applying to the parts of a unit of evolution. It is often supposed that this must be precluded by a restriction on laws that they make no reference to any spatio-temporally specific individual. But the motivation for this restriction is obscure. Kepler's laws, which refer to a mere handful of celestial objects, were once the best laws of nature going. They are not, of course, exactly true, but I suppose they might have been, and anyhow the laws that impress us most are not exactly, or even roughly, true most of the time (Cartwright, 1983). There is, on the other hand, an obvious reason from my perspective on the matter why we should not expect very reliable or fundamental laws about the members of species. This is simply that species are distinguished primarily for the purposes of classification, not generally for deeper theoretical reasons. If species are divorced to some degree from biological theory, then they will perhaps be equally distanced from laws. But then it is the vast diversity of biological phenomena that forces us to accept a classificatory system divorced from theory. So it may be that species provide the subject matter for laws as exact and fundamental as are available for biology at the organismic level.

My main point about these arguments, however, is that they start from the assumption that species are what evolve, that they are the units of evolution. And this is what I want to deny. I do not deny, on the other hand, that the things that evolve are historically specific individuals. If these are not species, what are they? The most plausible answer, and ultimately the one I take to be correct, is that the units of evolution are lineages. Lineages in general are sequences of entities related by ancestry and descent. The relevant entities in this case are populations, or groups of interconnected populations. Populations are themselves, I take it, individuals, and they are the temporal parts of other individuals, lineages. ${ }^{9}$ The general motivation for this suggestion is that evolutionary change is thought of as accumulating within lineages as it is passed from ancestors to descendants. Lineages should be distinguished from the technical concept of a clade since clades, but not lineages, are generally defined as including all the descendants of a specified ancestral popu-

\footnotetext{
${ }^{9} \mathrm{~A}$ useful discussion of the concept of a lineage is provided by de Queiroz (1999), though in the context of an argument I reject, that species are segments of lineages.
} 
lation. A clade, therefore, may contain distinct branches that are no longer a part of the same unit of evolution. There is no reason to think of a lineage, on the other hand, as ceasing to exist because a side-lineage achieves the status of an independent and distinct lineage.

It would be nice if the biological world divided up into fully distinct and internally fully coherent lineages. But of course it is just the failure of this occurrence that provided a fundamental obstacle to the attempt to assimilate units of classification to units of evolution. Typically we should expect to find ever smaller units of evolution-demes, temporarily isolated populations, etc.-within units of evolution, and in groups of organisms for which horizontal gene introgression is common, attempts to isolate the largest units of evolution will be to some degree arbitrary. This may be regrettable, but since the question what are the units of evolution is intended as properly ontological, in answering it we must attempt to record the actual state of things as accurately as possible. For the purposes of specific evolutionary investigations a degree of pluralism may well be appropriate. For some theoretical purposes it will be most useful to treat large, and perhaps only partially coherent, lineages as the units of evolution. For other purposes one might need to consider the properties of numerous and short-lived units. At any rate, whether we are considering major branches of the phylogenetic tree, local populations of particular organisms, or clones of bacteria maintaining their integrity for minutes or seconds, in so far as these are conceived as units of evolution, they are appropriately thought of as individuals.

\section{Some Consequences}

In a sense what I have been arguing is primarily a semantic point, though none the worse for that, I take it. However, a little more should perhaps be said about the rationale for the semantic decision here being advocated with regard to the word 'species'. Kim Sterelny (1999, p. 123) writes: 'we have to choose between the ideas that all organisms are members of some species and that being a species is an important biological property, for just as the organism as a grade of biological organization had to be invented, so did the species'. The first part of Sterelny's remark perfectly summarises my conclusions about the impossibility of coincidence between the units of evolution and the units of classification. But whereas I take the first option, on the grounds noted above that a system of classification must be comprehensive, Sterelny opts for the second. Sterelny argues that the biologically interesting level of organisation that corresponds, sometimes, to the traditional species, is a metapopulation consisting of an ecologically variable mosaic of populations. The choice Sterelny presents arises because, unsurprisingly, many kinds of organisms are not found in such biologically interesting metapopulations. But this makes Sterelny's semantic decision a bizarre one. On the one hand he offers us a term, 'metapopulation', that adequately captures the allegedly biologically interesting level of organisation. If this is not specific enough, one could be invented- 
'ecometapopulation', perhaps. On the other hand we have a term that has been used for several millennia to refer to the basic unit of biological classification. Recognising that in some cases the group of organisms distinguished by a traditional classificatory term coincides with a biologically interesting evolutionary individual strikes me as a very bad reason for coopting the term 'species' to refer solely to such individuals. So I enlist Sterelny as a hostile witness in support of my claim that we must give up the idea that being a species is an important biological property.

This raises a more general philosophical issue. It is often assumed, and I have made just this assumption myself, that classification in science must go hand in hand with theory. Progress in chemistry, for instance, is immediately reflected in improved classification of material stuffs, and modern chemistry is enshrined in the periodic table of the elements, both a scheme of classification and a vehicle for a good deal of chemical theory. Pure chemicals are the best surviving candidates for the traditional conception of natural kinds demarcated by the common possession of real essences. And something similar may be seen at the other end of the spectrum of organisational complexity. If Marx's social theories, for instance, have merit, then it should be theoretically illuminating-or anyhow should once have been-to classify people as proletarians and bourgeois. But of course no one thinks that all proletarians share a common real essence. What they share is what the theory says they share, a relation to the means of production. If the theory is a good one it will be useful for certain purposes to classify people by their relation to the means of production. But for other purposes it will be more relevant to speak of their nationality, their gender, the precise industry in which they work, or many other things. Perhaps chemistry is a simple enough science that one system of classification can be suited to all theoretical enquiries, but this is patently not the case for people. In fact for the human sciences there is no general taxonomy. The question what kind does that human belong to is meaningless without further context. There are, perhaps, people who think there is a clear answer to such questions, namely racists. But their views have thankfully been discredited, if by no means abandoned.

A view such as Sterelny's treats biology as in certain respects like the human sciences. It assumes that there is some theoretical project towards which the classification of organisms into species is directed, and that this project is the formulation of the best possible account of evolution. Since only certain species-like groups of organisms turn out to play a coherent role in the evolutionary process, only certain organisms belong to species. Similarly if there were wild people living in the woods off berries and roots a Marxist might say that they bear no relation to the means of production and thus belong to no social class. But the analogy between the biological and the human sciences breaks down here: in biology there is an important role for a general classification. As I have noted above, many different concerns, both theoretical and practical, generate an interest in the classification 
of organisms. But unlike the situation generally supposed to obtain in chemistry, there is no uniform theoretical perspective that will generate such a classification.

It appears, then, that the relation between theory and taxonomy is diverse. In the physical sciences the two are tightly yoked together by virtue of the existence of something close to traditional natural kinds; in the human sciences, paradoxically, they are also yoked together, this time by the fact that there are many different classifications relative to many different theoretical perspectives, and that there is no need for a general taxonomy. But in biology there are no such traditional natural kinds, many theoretical perspectives, but a massive diversity of highly diverse kinds. This situation forces classification to lead a life of its own, sometimes tightly connected to important theoretical perspectives, sometimes not.

One main reason why it is thought desirable that classification and theory should be closely connected is that we hope to discover laws about the kinds of things distinguished by our taxonomies. Indeed where the relation is most intimate, as in both the examples from chemistry and the examples from the human sciences, taxonomies are developed precisely in relation to laws that are discovered, or at least proposed, about members of kinds. Indeed, the possibility of formulating such laws might well have been added to my earlier list of desiderata for a classification. This brings up again the issue of whether there are laws about the members of biological species. This is certainly too large an issue for me to address in any detail here; for one thing, the question of what constitutes a scientific law, or a law of nature, is a thoroughly vexed one. What can safely be said is that the partial divorce of taxonomy from theory in biology provides one clear reason why we cannot expect laws of any fundamental importance to govern the behaviour of members of species. Perhaps the sheer number of different species is another such reason; laws referring to any one, unless perhaps our own, will be of only the most marginal importance. On the other hand, given that homogeneity is an important desideratum of the taxa generated by a classification, we should certainly expect there to be modest but fairly reliable generalisations about the members of species. And indeed the unduly maligned domain of natural history is largely the domain of such generalisations. I have no axe to grind about whether some or all of these should be accorded the honorific title of laws. Reasons for scepticism about such laws are often grounded in the assumption that species are units of evolution, and therefore subject to diachronic change and synchronic variability. If bacteria, for example, were to be classified instead in terms of morphology or ecological role, these arguments might be less compelling. But it would still be the case that the fact that we are concerned with only a minute fraction of the organic world will render the generalisations about a particular species of extremely local significance, and seldom if ever of deep theoretical import.

Let me finally say something about the question of realism. In earlier work (Dupré, 1993) I have insisted that despite the pluralism of biological classification, there is no reason to doubt that the kinds thus promiscuously distinguished should 
nevertheless have some reality, and indeed qualify, if in a sense rather weaker than the traditional one, for the status of natural kinds. I continue to maintain that there is no reason why the account of species currently offered should preclude their being modestly natural kinds. ${ }^{10}$ However the recognition of classification as the predominant driving force behind the distinction of species leads me to moderate my earlier promiscuous realism about biological kinds with a more promiscuous metaphysics. Often there are ways of classifying organisms in ways that correspond to modestly natural kinds, but often there may not be. Even in the latter casebacteria, brambles, perhaps-classification must go on. So I am inclined to say that some species are real natural kinds, but many are not.

\section{Conclusion}

The assumption that species must be units of evolution is undoubtedly related to the extent to which the figure of Darwin and his decisive contribution to biology dominates the discipline. As I quoted earlier, 'Nothing in biology makes sense apart from the theory of evolution' (Dobzhansky, 1973). I conclude with two comments about this remark. First, I am inclined to argue that the position I am defending reflects a further assimilation of Darwin's insights. It is often noted that Darwin put an end once and for all to the Aristotelian tradition of seeing biological species as natural kinds determined by real essences (Hull, 1965). This naturally led to a profound concern with the question: What, then, are species? Naturally, too, biologists and philosophers have tried to find an answer in the terms of the Darwinian paradigm. Darwin offered an account of the origin of biological diversity, and it has naturally been assumed that that was an account of the origin of species, where species would turn out to be something like what the Aristotelian perspective had assumed. Such an assumption was perhaps an essential stage in the evolution of our conception of biological diversity. But post-Darwinian biology has increasingly revealed that in many parts of the biological world there really are nothing like traditional species at all. Evolution has generated highly diverse patterns of diversity, some of which involve divisions similar to, or even coextensive with, what have previously been considered species, but some of which do not. Perhaps Darwin's work should have been entitled The Nonexistence of Species, except that no one would have had any reason to take such a work seriously. But even if there are no species in the Aristotelian sense, and no species in the sense of real units in nature reliably produced by the evolutionary process, we still have good reasons to impose some taxonomic order on the biological world. And in view of the picture of the biological world that has developed from Darwin's theory, this process can only be achieved with a methodology that is pragmatic, pluralistic, and sometimes frankly nominalist.

\footnotetext{
${ }^{10}$ One account of what might constitute them as natural kinds is that of Boyd (1999) and Wilson (1999).
} 
My second point is related to the first, but is slightly less respectful of the Darwinian hegemony. Though I have no doubt that every domain of biology is populated by organisms that evolved, there are parts of biology upon which evolution sheds less light than is often assumed. One of these, far removed from the present topic, is human behaviour. No doubt we evolved, and in a broad sense so did our behaviour. But the thesis that this fact is the key to understanding human behaviour has generally, and is currently, generating conclusions that range from the banal to the ridiculous. Parts of taxonomy may well also turn out to be areas where Darwin's insights shed less light than is often expected. David Nanney, an expert on the protozoans, a fascinating and bizarre group of organisms noted for their tendency to provide exceptions to widely assumed biological generalisations, has lucidly described the extraordinary difficulties in providing a taxonomy of an important groups of these organisms, the Tetrahymena, in the light of evolutionary principles. He remarks:

the earlier refusal of protozoologists to designate cryptic species by Latin binomials led some evolutionists to suppose that protozoologists do not understand evolutionary biology. Protozoologists, on the other hand, suspect that evolutionary geneticists do not always understand that taxonomy serves clients other than evolutionists. Different terms may be needed in different contexts (Nanney, 1999, p. 99).

It is part of the moral of this paper that we show no disrespect to Darwin when we recognise that sometimes there is more to biology than just the fact that organisms evolved.

\section{References}

Boyd, R. (1999) 'Homeostasis, Species, and Higher Taxa', in R. A. Wilson (ed.), Species: New Interdisciplinary Essays (Cambridge, MA: MIT Press), pp. 141-185.

Cartwright, N. (1983) How the Laws of Physics Lie (Oxford: Oxford University Press).

De Queiroz, K. (1999) 'The General Lineage Concept of Species and the Defining Property of the Species Category', in R. A. Wilson (ed.), Species: New Interdisciplinary Essays (Cambridge, MA: MIT Press), pp. 49-89.

Dobzhansky, T. (1973) 'Nothing in Biology Makes Sense Except in the Light of Evolution', American Biology Teacher 35, 125-129.

Dupré, J. (1993) The Disorder of Things: Metaphysical Foundations of the Disunity of Science (Cambridge, MA: Harvard University Press).

Dupré, J. (1999) 'On the Impossibility of a Monistic Account of Species', in R. A. Wilson (ed.), Species: New Interdisciplinary Essays (Cambridge, MA: MIT Press), pp. 3-22.

Ereshefsky, M. (1992) The Units of Evolution (Cambridge, MA: MIT Press).

Ehrlich, P. R. and Raven, P. H. (1969) 'Differentiation of Populations', Science 165, 12281232. (Reprinted in Ereshefsky, 1992).

Ghiselin, M. T. (1997) Metaphysics and the Origin of Species (Albany, NY: SUNY Press).

Hull, D. L. (1965) 'The Effect of Essentialism on Taxonomy: Two Thousand Years of Stasis', British Journal for the Philosophy of Science 15, 314-326; 16, 1-18. (Reprinted in Ereshefsky, 1992).

Magnus, D. (1998) 'Evolution Without Change in Gene Frequencies', Biology and Philosophy 13, 255-261.

Mayr, E. (1996) 'What Is a Species, and What Is Not', Philosophy of Science 63, 262-277.

Mishler, B. D. and Donoghue, M. J (1982) 'Species Concepts: A Case for Pluralism', Systematic Zoology 31, 491-503. (Reprinted in Ereshefsky, 1992). 
Nanney, D. (1999) 'When is a Rose?: The Kinds of Tetrahymena', in R. A. Wilson (ed.), Species: New Interdisciplinary Essays (Cambridge, MA: MIT Press), pp. 93-118.

Ruse, M. (1987) 'Biological Species: Natural Kinds, Individuals, or What?', British Journal for the Philosophy of Science 38, 225-242. (Reprinted in Ereshefsky, 1992).

Stebbins, G. L. (1987) 'Species Concepts: Semantics and Actual Situations', Biology and Philosophy 2, 198-203.

Sterelny, K. (1999) 'Species as Ecological Mosaics', in R. A. Wilson (ed.), Species: New Interdisciplinary Essays (Cambridge, MA: MIT Press), pp. 119-138.

Templeton, A. R. (1989) 'The Meaning of Species and Speciation: A Genetic Perspective', in D. Otte and J. A. Endler (eds), Speciation and its Consequences (Sunderland, MA: Sinauer), pp. 3-27. (Reprinted in Ereshefsky, 1992. Page references are to this reprint.)

Van Valen, L. (1976) 'Ecological Species, Multispecies, Oaks', Taxon 25, 233-239. (Reprinted in Ereshefsky, 1992).

Wilson, R. A. (1999) Species: New Interdisciplinary Essays (Cambridge, MA: MIT Press). 